\title{
Low Grade Ovarian Serous Adenocarcinoma
}

National Cancer Institute

\section{Source}

National Cancer Institute. Low Grade Ovarian Serous Adenocarcinoma. NCI Thesaurus. Code C105556.

A slow-growing serous adenocarcinoma that arises from the ovary. It usually originates from borderline neoplastic processes or adenofibromas. It is characterized by the presence of low grade cytologic features and infrequent mitotic figures. 